\title{
Is There an Advantage to Knotless Barbed Suture in TKA Wound Closure? A Randomized Trial in Simultaneous Bilateral TKAs
}

\author{
Alexander P. Sah MD
}

Received: 2 September 2014/Accepted: 14 January 2015 / Published online: 29 January 2015

(C) The Association of Bone and Joint Surgeons $\mathbb{R} 2015$

\begin{abstract}
Background Effective wound closure is critical to minimizing wound complications and withstanding the forces associated with early knee motion after TKA. Barbed sutures allow for knotless fixation, have been used successfully in other specialties, and may provide for more even distribution of tension along the length of the incision; however, data regarding unidirectional barbed sutures from randomized trials have raised important concerns about their use. Bidirectional barbed sutures offer a potential alternative, but have not been studied extensively in orthopaedic surgery.

Questions/purposes Using a prospective, randomized, within-patient controlled study design I compared wound closure performed with bidirectional barbed sutures in one

The author certifies that he, or a member of his immediate family, has no funding or commercial associations (eg, consultancies, stock ownership, equity interest, patent/licensing arrangements, etc) that might pose a conflict of interest in connection with the submitted article.

All ICMJE Conflict of Interest Forms for authors and Clinical Orthopaedics and Related Research ${ }^{\mathbb{R}}$ editors and board members are on file with the publication and can be viewed on request.

Clinical Orthopaedics and Related Research ${ }^{\mathbb{B}}$ neither advocates nor endorses the use of any treatment, drug, or device. Readers are encouraged to always seek additional information, including FDA-approval status, of any drug or device prior to clinical use. Each author certifies that his or her institution has approved the human protocol for this investigation, that all investigations were conducted in conformity with ethical principles of research, and that informed consent was obtained.
\end{abstract}

\section{A. P. Sah $(\bowtie)$}

Department of Orthopedics, Institute for Joint Restoration,

Washington Hospital Healthcare System, 2000 Mowry Avenue,

Fremont, CA 94538, USA

e-mail: asah07@gmail.com knee of bilateral TKAs performed under the same anesthetic with those performed with standard sutures in the other knee to determine whether the barbed suture was associated with (1) faster closure times; (2) fewer intraoperative suture issues, such as needle sticks or suture breakage, and fewer postoperative wound complications; (3) no detrimental effect on clinical outcomes, including knee ROM and Knee Society scores; and (4) lower total operative cost, considering suture material cost and operating room time savings.

Methods Between 2011 and 2012, 50 consecutive patients meeting prespecified inclusion criteria with simultaneous bilateral TKAs had deep and superficial closures performed using interrupted and running standard sutures in one randomly assigned knee, and running knotless bidirectional barbed sutures in the other knee. The barbed suture is US FDA-approved for soft tissue approximation wherever absorbable sutures are appropriate. Intraoperative suture issues and the number of sutures used were recorded at the time of wound closure. Suture cost was compared between the standard and barbed sutures and measured against the operative time cost, as estimated per minute saved. Patients were followed postoperatively at 2, 6, and 12 weeks, and 1 year. Outcomes assessed included detailed operative and tourniquet time, knee ROM, Knee Society scores, postoperative complications, use of antibiotics, and any subsequent surgical interventions. These outcomes were assessed at each visit except for Knee Society scores which were collected at the 12-week and 1-year evaluations. All patients completed followups up to the final evaluation at 1 year.

Results Mean wound closure time was 4.7 minutes less using barbed sutures (SD, $\pm 2.8 ; 95 \% \mathrm{CI},-5.5$ to -3.7 ; $\mathrm{p}<0.001)$, average $16.1(\mathrm{SD}, \pm 2.2)$ versus $11.4(\mathrm{SD}, \pm$ 2.2) minutes for the standard versus barbed suture types, 
respectively. Overall tourniquet time was not different at 78.7 minutes (SD, \pm 11.1 minutes) versus 74.9 minutes (SD, \pm 10.1 minutes), respectively $(\mathrm{p}>0.1)$. There were no intraoperative clinical issues, such as provider or patient injury, using either suture. There were no needle disengagements or suture breakages with barbed-suture closure; five episodes of premature disengagement of the suture from the needle and three suture breakages were observed with standard closures $(\mathrm{p}<0.005)$. There were no postoperative wound dehiscences or disruptions of the arthrotomy closure with either closure technique. Final ROM was not different with the numbers available (barbed-suture group mean, $126.7^{\circ} \pm 6.9^{\circ} \mathrm{SD}$ vs standardsuture group mean, $125.6^{\circ} \pm 7.0^{\circ} \mathrm{SD} ; \mathrm{p}=0.4,95 \% \mathrm{CI}$, -3.77 to 1.73 ) between patient groups at 1 year. There were no differences with the numbers available in 1-year Knee Society knee scores (barbed mean, $92.8 \pm 6.69$ SD vs standard mean, $93.3 \pm 6.2 \mathrm{SD} ; \mathrm{p}=0.6,95 \% \mathrm{CI},-1.97$ to 3.36). Considering suture material cost against time savings in operating room time, there was a cost savings of mean USD 175 per case when using barbed suture.

Conclusions In this randomized controlled trial, I found knotless bidirectional barbed suture to be more efficient in terms of closure time and lower in direct operative cost than conventional suture material, while showing no difference in terms of Knee Society knee scores, ROM, or wound appearance with the numbers available. Future studies with larger numbers will be needed to compare overall costs of care and to detect uncommon complications that might arise, although none were observed in this small series.

Level of Evidence Level I, therapeutic study.

\section{Introduction}

Less-invasive surgical techniques have led to modifications in surgical instruments and techniques implemented with the intent of minimizing soft tissue trauma and potentially leading to faster patient recovery. However, in the process, relatively less focus may have been spent on the importance of optimizing wound closure. With TKAs particularly, a quality closure is critical to minimizing wound complications and withstanding forces across the incision during early knee motion after surgery.

Compared with standard sutures, the design of bidirectional barbed sutures allows for knotless fixation and provides for more even distribution of tension along the entire length of the incision [8]. Evidence from cadaver knee studies suggests that bidirectional barbed sutures may provide a more watertight closure [6] and evidence from biomechanical testing suggests that TKAs closed with bidirectional barbed sutures are more resistant to failure than those where interrupted standard sutures have been used [12].

First introduced in 2007, the knotless, bidirectional, barbed suture is used by surgeons from numerous specialties, including plastic and reconstructive surgery, urology, obstetrics, and laparoscopic gynecology [8]; however some skepticism regarding the risk or benefit profile of the suture technology in orthopaedic applications persists. This may be partly attributable to a statistically significantly higher frequency of postoperative wound complications reported in two large studies $(n=694$ patients) in which closures were performed using the unidirectional barbed suture, V-Loc ${ }^{\mathrm{TM}}$ (Covidien, Mansfield, MA, USA) [1, 7]; or the three reported occurrences of extensor mechanism repair failure with the bidirectional barbed suture [13]. Most clinical data for the bidirectional barbed suture have been collated from retrospective patient chart reviews [2, 3, 9-11], with only one large multicenter, prospective study with final skin closure method variation reported to date [3].

I therefore designed a prospective study in which patients undergoing simultaneous bilateral TKAs served as their own controls, with closure of all layers of one knee performed with standard sutures and closure of the other knee performed with knotless bidirectional barbed sutures. The two closure approaches were compared in terms of (1) closure times; (2) intraoperative suture issues, such as needle sticks or suture breakage, and postoperative wound complications; (3) clinical outcomes including knee ROM and Knee Society scores; and (4) total operative cost, considering suture material cost with operating room time savings.

\section{Methods}

I performed a single-center, prospectively designed, controlled study. Approval from the institutional review board (IRB) was obtained before study commencement. The study was conducted between January 2011 and December 2012 by one surgeon (APS), with a physician assistant (AG), at a community hospital with a comprehensive joint arthroplasty program.

Fifty consecutive patients who met the indications for simultaneous bilateral TKAs were invited and all subsequently agreed to participate in the study. Exclusion criteria included prior surgical incisions in the area of the planned surgical approach. No patients were excluded or withdrawn from the study. The study population consisted of 21 men and 29 women with a mean age of 68.1 years (SD \pm 8.5 years). BMI for the patients was mean of 30.1 $(\mathrm{SD}, \pm 4.6)$. All study patients had a diagnosis of 
osteoarthritis. Prior procedures included five knee arthroscopies. No patients were lost to followup.

Patients served as their own controls, and knees were randomly assigned to receive the barbed suture by computer generation to minimize any effect of surgeonhandedness and to blind the clinician at the time of woundappearance evaluation. A medial parapatellar approach was used in all procedures. The arthrotomy was closed by the surgeon and physician assistant together, regardless of suture type used. Suturing of the more superficial layers was performed by the same physician assistant in all cases. Standard closures were performed using eight interrupted Number 1 Vicryl $^{\mathrm{TM}}$ pop-off sutures (Ethicon Inc; Somerville, NJ, USA) for the retinaculum, followed by four to six Number 2-0 Vicryl ${ }^{\mathrm{TM}}$ sutures (Ethicon Inc) to approximate the deep-intermediate layer, then a Number 2-0 Monocryl ${ }^{\circledR}$ suture (Ethicon Inc) for a running subcutaneous closure, and finally a Number 3-0 Monocryl ${ }^{\mathbb{R}}$ (Ethicon Inc) for a running subcuticular closure. Barbed closures were performed using comparable sizes (increased by one suture size by product recommendation) and types of the bidirectional Quill ${ }^{\mathrm{TM}}$ Knotless Tissue-Closure Device. For the barbed group, Number 2 Quill $^{\mathrm{TM}}$ was used in a running fashion to close the retinaculum. To approximate the deepintermediate layer, four to six Number 2-0 Vicryl $^{\mathrm{TM}}$ sutures (Ethicon Inc) were used, similar to the standard group. The subcutaneous and subcuticular layers then were closed with a running knotless barbed 2-0 Monoderm $^{\mathrm{TM}}$ (Angiotech; Surgical Specialties Corp; Reading, PA, USA). Barbed sutures were placed by starting from the midpoint of the wound layer and proceeding simultaneously toward the opposing wound ends. Tension was applied evenly by using traction at distal ends of the barbed suture. At each wound end, barbed closures were secured with two backtrack throws of the suture and were knotless. The tourniquet was deflated after wound closure, as a sterile dressing was placed.

Patient baseline data were compiled at the time of screening and/or on the day of the TKAs. Suture type and size for each knee, tourniquet time, total operative time, time for wound closure (defined as the start of first suture placed in the arthrotomy to the final stitch of skin closure), and any intraoperative suture issues (eg, needle sticks, suture breakage) were entered in the surgical record. Physical therapy began on postoperative Day 1 with ambulation and ROM exercises. Patients were followed postoperatively at 2,6 , and 12 weeks, and at 1 year. Wound sites were evaluated and charted daily until hospital discharge, and then at each postoperative visit. Surgical sites were evaluated by the same physician assistant and the surgeon at each visit. Knee ROM was evaluated at each visit. Postoperative complications, such as wound dehiscence, disruption of the arthrotomy closure, infection, drainage, cellulitis, suture irritation, and suture abscess were recorded in the patient's case record. Use of oral antibiotics for wound concerns and any subsequent surgical interventions also were recorded. The Knee Society scores were collected preoperatively, at 3 months, and at 1 year.

Cost analysis was performed based on the hospital cost of suture material and operating room time. Operating room time per minute at my institution varies based on numerous individual case factors and averages USD 48 per minute (range, USD 32-64/minute), similar to published costs of 100 United States hospitals which averaged USD 62 per minute (range, USD 22-133/minute) [5].

Data were analyzed using commercially available software (GraphPad; GraphPad Software, San Diego, CA, USA). Fisher's exact and chi-square tests were performed, using a threshold for statistical significance of $p$ less than 0.05 . A post hoc power analysis was performed for the primary study question (closure time) and 50 paired knees provided $80 \%$ power to reveal a 1.2 -minute difference between suture types used in closure time.

\section{Results}

Barbed running sutures allow faster wound closure times compared with standard sutures. Time of wound closure was a mean of 4.7 minutes (SD, \pm 2.8 minutes; $95 \% \mathrm{CI},-5.5$ to $-3.7 ; \mathrm{p}<0.001$ ) faster with barbed suture, mean 11.4 minutes (SD, \pm 2.2 minutes) versus 16.1 minutes $(\mathrm{SD}, \pm 2.2 \mathrm{~min}$ utes) for barbed versus standard sutures, respectively. Overall tourniquet time was 78.7 minutes ( $\mathrm{SD}, \pm 11.1$ minutes) versus 74.9 minutes $\quad(\mathrm{SD}, \pm 10.1$ minutes), respectively $(\mathrm{p}=0.11)$.

Barbed sutures showed benefits of fewer suture handoffs and suture failures, but was not different in terms of wound complications with the numbers available. The number of suture handoffs between scrub technicians and the surgeon was less with barbed sutures with seven to nine sutures versus 14 to 16 passes with standard sutures $(\mathrm{p}<0.001)$. Despite this, there were no intraoperative clinical complications (provider needle sticks) using either suture type. There were no suture breakages or failures using the barbed suture, but five episodes of premature disengagement of the suture from the needle and three suture breakages during knot tying with the standard type suture were observed $(p<0.005)$. There were no actively draining wounds on discharge and no postoperative deep or superficial wound dehiscence or disruption of closure with use of either suture type. There were three suture abscesses with traditional sutures and none with barbed sutures $(\mathrm{p}=0.24)$. There was one additional episode of suture spitting through the skin in the standard suture group ( $p>0.5)$. No antibiotics 
were used and no surgical interventions were required in either group.

Clinical knee assessment in terms of ROM and Knee Society knee scores were not different between knees with the numbers available. Preoperative Knee Society knee scores were not different with the numbers available (barbed mean, $55.8 \pm 16.8 \mathrm{SD}$ vs standard mean, $55.7 \pm 15.14 \mathrm{SD} ; \mathrm{p}=0.9,95 \% \mathrm{CI},-7.26$ to 7.21$)$. Before discharge, ROM in both knees of the patients averaged greater than $99^{\circ}$ (barbed mean, 100.2 \pm 7.59 SD vs standard mean, $99.8 \pm 15.43 \mathrm{SD} ; \mathrm{p}=0.8,95 \% \mathrm{CI},-4.47$ to 5.28). There were no differences with the numbers available in wound complications with physical therapy and ambulation begun the day after surgery. No knee manipulations were required. ROM remained similar throughout postoperative visits, and final ROM at 1 year was not different with the numbers available between knees (barbedsuture group mean, $126.7^{\circ} \pm 6.9^{\circ} \mathrm{SD}$ vs standard-suture group mean, $125.6^{\circ} \pm 7.0^{\circ} \mathrm{SD} ; \mathrm{p}=0.4,95 \% \mathrm{CI},-3.77$ to 1.73). Knee Society scores also were not different at 3 months (barbed mean, 89.2 \pm 7.27 SD vs standard mean, $90.4 \pm 5.79 \mathrm{SD} ; \mathrm{p}=0.37,95 \% \mathrm{CI},-3.84$ to 1.43 ) or 1 year (barbed mean, $92.8 \pm 6.69 \mathrm{SD}$ vs standard mean, $93.3 \pm 6.2 \mathrm{SD} ; \mathrm{p}=0.6,95 \% \mathrm{CI},-1.97$ to 3.36$)$ with the numbers available. The delta change from preoperative to final followup for Knee Society knee scores was not different (barbed mean, $38.0 \pm 16.6 \mathrm{SD}$ vs standard mean, $38.1 \pm 18.59 \mathrm{SD} ; \mathrm{p}=0.9,95 \% \mathrm{CI},-7.88$ to 8.03$)$.

While the material costs more than standard counterparts, barbed suture saves cost by allowing faster wound closures and reducing operating room time. Cost of the suture material was USD 32 in the standard group. Pop-off Number 1 Vicryl $^{\mathrm{TM}}$ sutures, in a pack of eight, are used to maximize speed of wound closure when using standard suture (USD 12). Vicryl ${ }^{\mathrm{TM}}$ Number 2-0 pop-off sutures in a pack of eight cost USD 12. A Number 2-0 Monocryl ${ }^{\circledR}$ suture (USD 2.5) and Number 3-0 Monocryl ${ }^{\circledR}$ suture (USD 5.6) are used to close the skin. The barbed-suture closure technique had a cost of USD 82. The Number 2 Quill $^{\mathrm{TM}}$ barbed suture costs USD 22, the Number 2-0 Vicryl ${ }^{\mathrm{TM}}$ pop-off sutures cost USD 12, and the Number 2-0 barbed suture (two used per case) costs USD 24 each. Operating time savings was based on the average 4.7 minutes faster closure time, which equaled USD 150 to 300 per case when barbed suture was used. Total savings per case, after including suture cost, with use of the barbed suture averaged USD 175 (range, USD 100-250). The current technique I use with barbed sutures requires use of only one Number 2-0 barbed suture to close the skin with a duallayer technique (one arm run in the deeper layer, the other subcuticular), thereby reducing the total cost to USD 58 and increasing total savings per case with barbed sutures when including the continued time savings.

\section{Discussion}

Effective wound closure is critical to minimizing wound complications and withstanding the forces associated with early knee motion after TKA. Barbed sutures allow for knotless fixation, but randomized studies on unidirectional barbed sutures have raised concerns about them [1, 7], and to my knowledge, there have been no randomized trials with bidirectional barbed sutures used for complete wound closure in TKAs; nonrandomized studies have methodologic shortcomings that limit the conclusions that can be drawn from them. By design, the current study of 50 simultaneous bilateral TKAs performed by the same surgeon (APS) allowed for direct within-patient comparisons of key outcomes while limiting potentially confounding factors, such as interpatient differences, and intersurgeon variability. Using this method, I hoped to answer whether knotless bidirectional barbed suture provided advantages in time and cost savings while maintaining excellent cosmetic and functional results compared with standard suture. Specifically, I asked whether the barbed device was associated with (1) faster closure times; (2) fewer intraoperative suture issues, including suture breakage and needle sticks, and postoperative wound complications; (3) no detrimental effect on clinical outcomes such as knee ROM or Knee Society scores; and (4) lower direct operative cost.

This study had numerous limitations. First, while reporting the experience of one surgeon eliminates multisurgeon variability, it also limits generalizability; complications of suture breakage or needle sticks may vary with surgeon experience using the barbed device. Second, only TKAs were evaluated, so generalizations regarding THAs or other orthopaedic procedures cannot be made, but typically knee wounds undergo early, high forces with rapid rehabilitation. Third, while bias could exist because the surgeon and physician assistant performed the wound evaluations, this process provided consistency in evaluation and the randomization process addressed possible bias. The randomization also eliminated the dominant handedness of the surgeon or physician assistant from affecting wound closure time. Further evaluation at my institution has shown similar time savings in subsequent hip and knee surgery wound closures. Finally, although post hoc power analysis showed a more than adequate sample size to answer my first question (time of closure), the relatively small sample size was underpowered to address whether the small number of suture issues (breakage and needle sticks) and superficial stitch abscesses differed significantly between the suture-type groups. Thus, I cannot comment on the safety of the barbed suture. However, my study does represent a relatively large group of unique within-patient controls, and the comparisons did not show a detriment with use of barbed suture. 


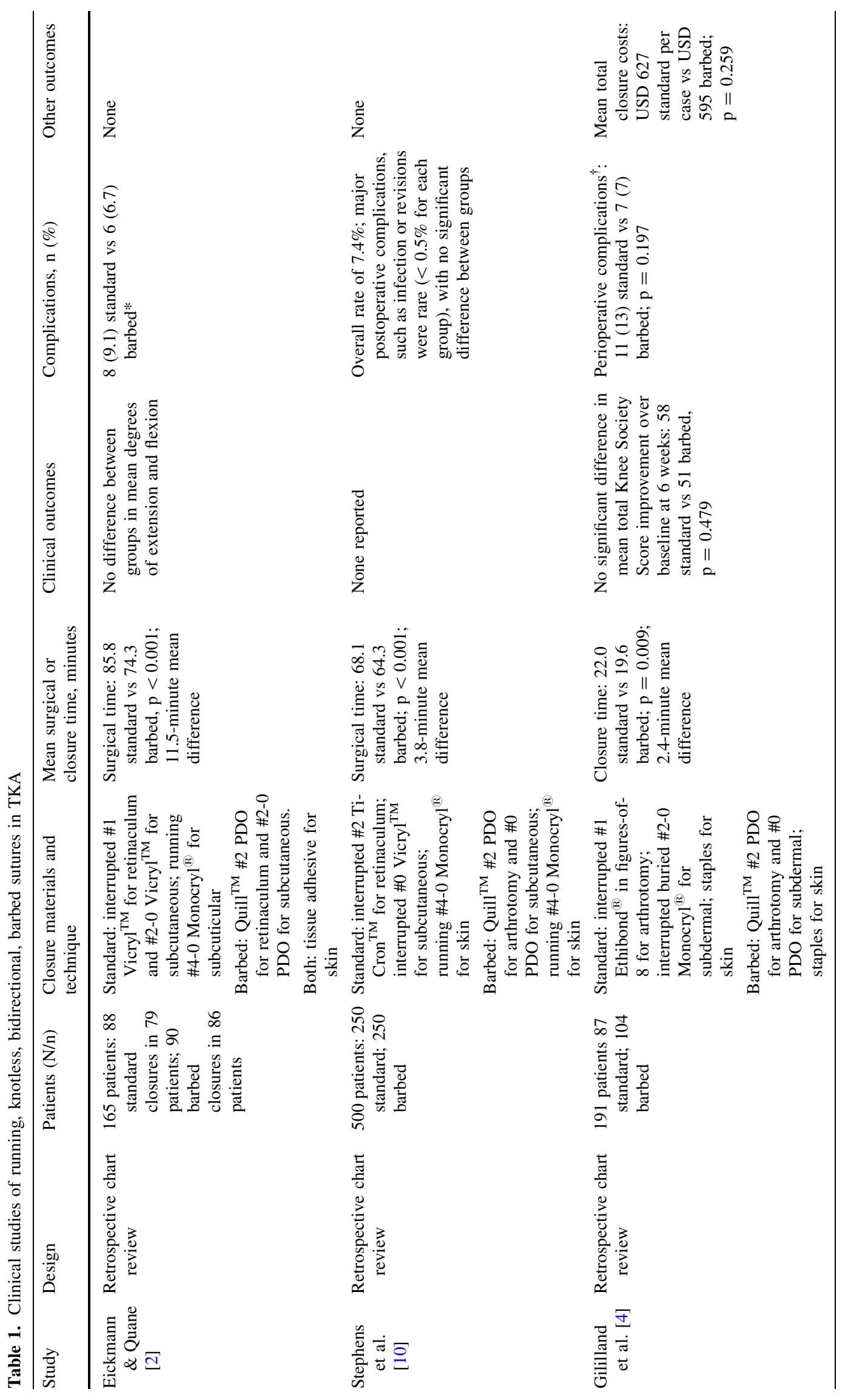




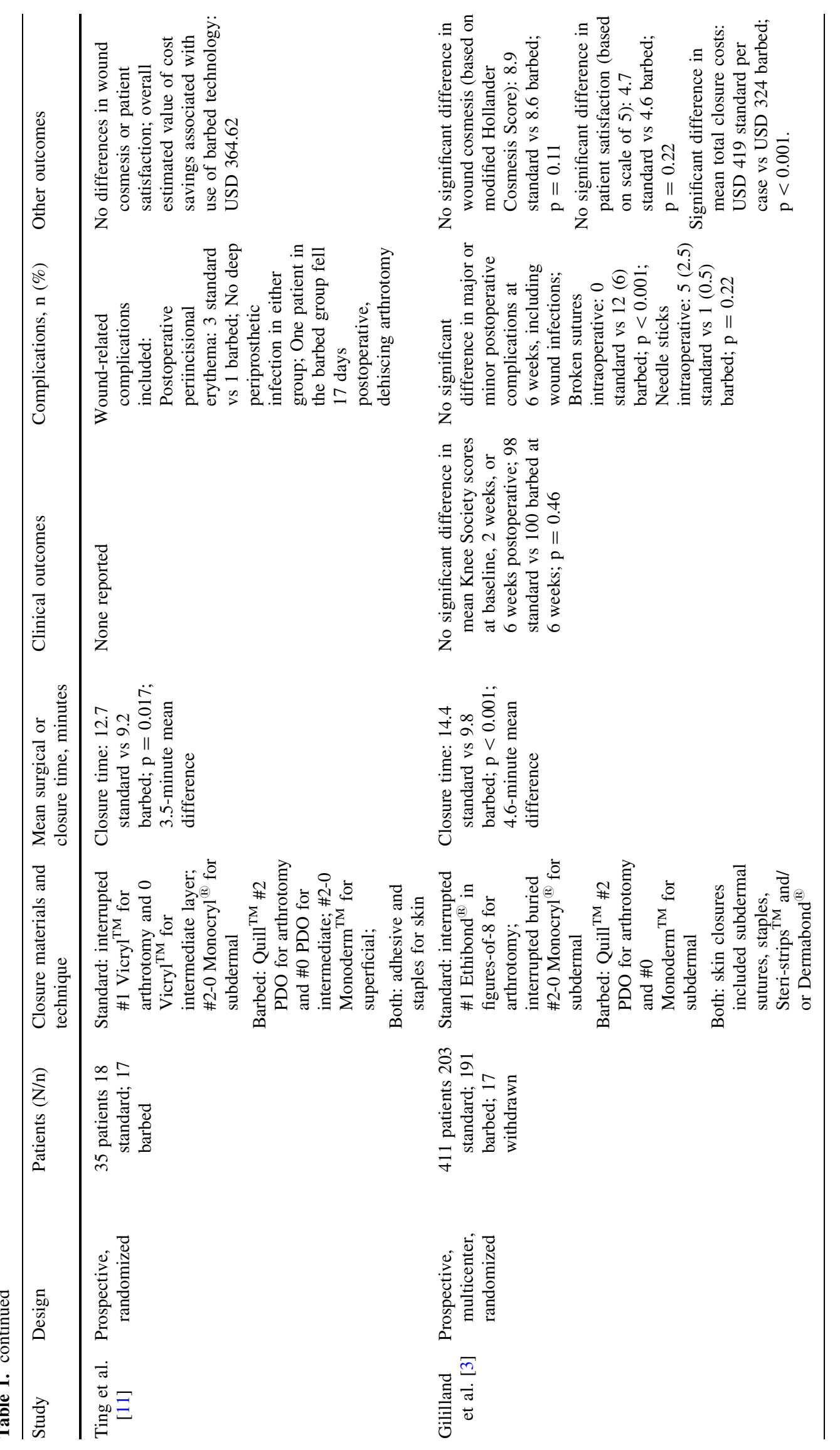




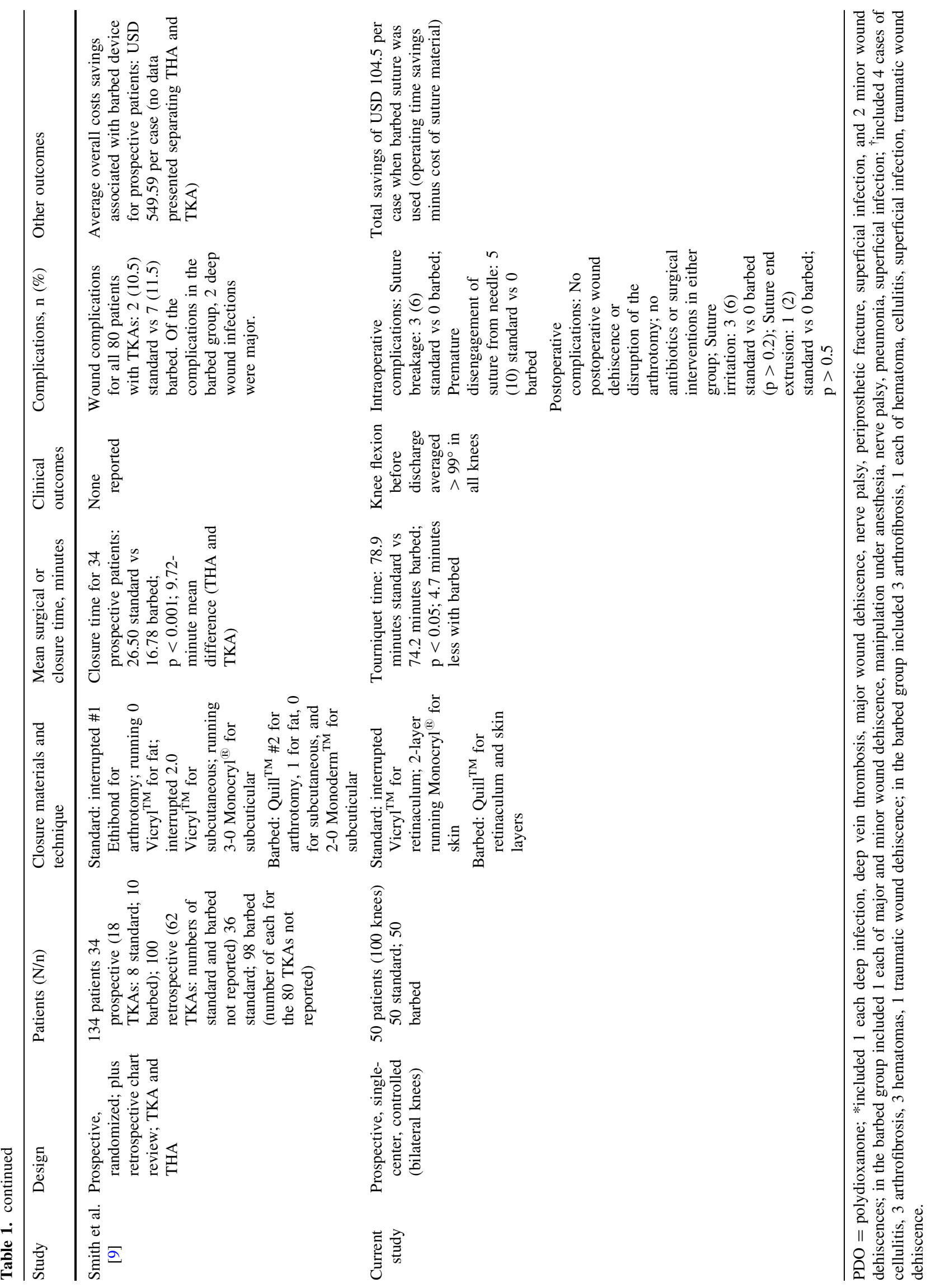


In the current study, barbed closures were associated with a mean savings of 5 minutes, which is in the range of 3 to 12 minutes reported in other studies with use of this device (Table 1) [2-4, 9-11]. Running knotless closures with barbed sutures consistently are reported to have shorter closure times than standard interrupted closure techniques [2-4, 8-10].

As with five of the six previously reported studies [2, 4, 9-11], no differences in intraoperative suture issues or postoperative wound complications were observed in the current study (Table 1). However, to detect differences in rare complications such as dehiscence or infection requires much larger studies than have been conducted to this point. Future systematic reviews and meta-analyses will be needed to pool results and evaluate differences in event rates for uncommon problems such as wound dehiscence or infections. Well-controlled randomized trials like the current study can help future meta-analyses evaluate this important set of safety questions. To date, the only difference observed in comparing standard with barbed sutures has pertained to intraoperative suture breakage [3]. In the large, prospective, multicenter study by Gililland et al. [3], 12 sutures were broken in the barbed closure group (explained as occurring during the learning curve), whereas none were broken in the standard closure group $(\mathrm{p}<0.001)$. They reported more needle sticks with standard sutures, but the occurrence was not statistically significant. In the current study, there were no needle sticks, but the number of times a suture was passed was greater in the standard suture group, potentially increasing the chance of injury to the surgeon or assistant performing suturing.

Quality wound closure is critical to resist the high tension of knee surgery wounds, especially with rapid recovery protocols. In the current study, I found that knee incisions closed with the barbed suture did not differ with the numbers available in Knee Society knee scores or final flexion from those closed with standard sutures. This result is consistent with those of previous reports in which no differences were observed between barbed and standard closure groups in mean degrees of extension and flexion [2], or in the mean total Knee Society knee scores at 6 weeks $[3,4]$. In the current study, clinical outcomes were excellent regardless of the wound closure method used.

The relative benefit of saving 5 minutes of operative time may not seem apparent at first. However, when suture costs and time savings are factored in, an overall costs savings of USD 100 to 250 per case with knotless barbed sutures was realized. This is comparable to what has been reported in other studies in which overall costs were calculated $[3,4,7,9,11]$. In those studies, barbed closures were associated with cost savings between USD 30 and
USD 550 per procedure, also in large part attributable to reduced operating time $[3,4,7,9,11]$.

In the current randomized controlled trial, I found knotless bidirectional barbed suture to be more efficient in terms of time and direct cost than conventional suture material, and no different in terms of Knee Society knee scores or ROM. More efficient wound closure may have benefits outside the cost of operating room time, including shorter time of wound exposure, potentially more efficient use of scrub personnel time during wound closure, and less risk of needle sticks while suturing or passing suture. Continued prospective evaluation of knotless barbed sutures in orthopaedic settings is justified and future metaanalyses of randomized trials such as this will help to compare overall costs of care and to detect uncommon complications that might arise.

Acknowledgment I thank Abigail Goetz PA (Department of Orthopedics, Institute for Joint Restoration, Washington Hospital Healthcare System, Fremont, CA, USA) for assisting with the surgical procedures and evaluations of the study patients.

\section{References}

1. Campbell AL, Patrick DA Jr, Liabaud B, Geller JA. Superficial wound closure complications with barbed sutures following knee arthroplasty. J Arthroplasty. 2014;29:966-969.

2. Eickmann T, Quane, E. Total knee arthroplasty closure with barbed sutures. J Knee Surg. 2010;23:163-168.

3. Gililland J, Anderson L, Barney J, Ross HL, Pelt CE, Peters CL. Barbed versus standard sutures for closure in total knee arthroplasty: a multicenter prospective randomized trial. J Arthroplasty. 2014;29:135-138.

4. Gililland JM, Anderson LA, Sun G, Erickson JA, Peters CL. Perioperative closure-related complication rates and cost analysis of barbed suture for closure in TKA. Clin Orthop Relat Res. 2012;470:125-129.

5. Macario A. What does one minute of operating room time cost? J Clin Anesthesia. 2010;22:233-236.

6. Nett M, Avelar R, Sheehan M, Cushner F. Water-tight knee arthrotomy closure: comparison of a novel single bidirectional barbed self-retaining running suture versus conventional interrupted sutures. J Knee Surg. 2011;24:55-60.

7. Patel RM, Cayo M, Patel A, Albarillo M, Puri L. Wound complications in joint arthroplasty: comparing traditional and modern methods of skin closure. Orthopedics. 2012; 35:e641-e646.

8. Paul M. Bidirectional barbed sutures for wound closure: evolution and applications. J Am Col Certif Wound Spec. 2009;1:51-57.

9. Smith EL, DiSegna ST, Shukla PY, Matzkin EG. Barbed versus traditional sutures: closure time, cost, and wound related outcomes in total joint arthroplasty. J Arthroplasty. 2014;29:283287.

10. Stephens S, Politi J, Taylor BC. Evaluation of primary total knee arthroplasty incision closure with the use of continuous bidirectional barbed suture. Surg Technol Int. 2011;XXI:199-203.

11. Ting N, Moric MM, Della Valle CJ, Levine BR. Use of a knotless suture for closure of total hip and knee arthroplasties: a prospective, randomized clinical trial. J Arthroplasty. 2012;27:17831788. 
12. Vakil JJ, O'Reilly MP, Sutter EG, Mears, SC, Belkoff SM, Khanuja HS. Knee arthrotomy repair with a continuous barbed suture: a biomechanical study. J Arthroplasty. 2011;26: 710-713.
13. Wright RC, Gillis CT, Yacoubian SV, Raven RB III, Falkinstein Y, Yacoubian SV. Extensor mechanism repair failure with use of bidirectional barbed suture in total knee arthroplasty. J Arthroplasty. 2012;27:1413.e1-e4. 